\title{
Rate and Power Allocation in Fading Multiple Access Channels
}

\author{
Ali ParandehGheibi, Atilla Eryilmaz, Asuman Ozdaglar, and Muriel Médard
}

\begin{abstract}
We consider the problem of rate and power allocation in a fading multiple-access channel. Our objective is to obtain rate and power allocation policies that maximize a utility function defined over average transmission rates. In contrast with the literature, which focuses on the linear case, we present results for general concave utility functions. We consider two cases. In the first case, we assume that power control is possible and channel statistics are known. In this case, we show that the optimal policies can be obtained greedily by maximizing a linear utility function at each channel state. In the second case, we assume that power control is not possible and channel statistics are not available. In this case, we define a greedy rate allocation policy and provide upper bounds on the performance difference between the optimal and the greedy policy. Our bounds highlight the dependence of the performance difference on the channel variations and the structure of the utility function.
\end{abstract}

\section{INTRODUCTION}

Dynamic allocation of communication resources such as bandwidth or transmission power is a central issue in multiple access channels in view of the time varying nature of the channel and interference effect. Most of the existing literature focuses on specific communication schemes such as TDMA (time-division multiple access) [1] and CDMA (code-division multiple access) [2], [3] systems. An exception is the work by Tse et al. [4], who consider the notion of throughput capacity for the fading channel with Channel State Information (CSI). That is the notion of Shannon capacity applied to the fading channel, where the codeword length can be arbitrarily long to average over the fading of the channel. The points on the boundary of the capacity region are attained by dynamically allocating the resources with the goal of maximizing a linear utility function.

In this paper, we consider the problem of rate and power allocation in a multiple access channel with perfect CSI. Contrary to the linear case in [4], we consider maximizing a general utility function of transmission rates over the throughput capacity region. Such a general concave utility function allows us to capture different performance metrics such as fairness or delay (c.f. Shenker [5], Srikant [6]). Our contributions can be summarized as follows.

A. ParandehGheibi is with the Laboratory for Information and Decision Systems, Electrical Engineering and Computer Science Department, Massachusetts Institute of Technology, Cambridge MA, 02139 (e-mail: parandeh@mit.edu)

A. Eryilmaz is with the Electrical and Computer Engineering, Ohio State University, OH, 43210 (e-mail: eryilmaz@ece.osu.edu)

A. Ozdaglar and M. Médard are with the Laboratory for Information and Decision Systems, Electrical Engineering and Computer Science Department, Massachusetts Institute of Technology, Cambridge MA, 02139 (e-mails: asuman@mit.edu, medard@mit.edu)
We first consider the case where channel statistics are known and power can be controlled at the transmitters. Owing to strict convexity of the capacity region, we show that the resource allocation problem for a general concave utility is equivalent to another problem with a linear utility. Hence, the optimal resource allocation policies are obtained by applying the results in [4] for the linear utility. Given a general utility function, the conditional gradient method is used to obtain the corresponding linear utility. Second, we consider the case where the transmitters do not have the power control feature and channel statistics are not known. In this case, a greedy policy which maximizes the utility function for any given channel state, is suboptimal. However, we can bound the performance difference between the optimal and the greedy policies. We show that this bound is tight in the sense that it goes to zero either as the utility function tends to a linear function of the rates or as the channel variations vanish.

Other than the papers cited above, our work is also related to the work of Vishwanath et al. [7] which builds on [4] and takes a similar approach to the resource allocation problem for linear utility functions. Other works address different criteria for resource allocation including minimizing the weighted sum of transmission powers [8], and considering Quality of Service (QoS) constraints [9]. In contrast to this literature, we consider the utility maximization framework for general concave utility functions.

The remainder of this paper is organized as follows: In Section II, we introduce the model and describe the capacity region of a fading multiple-access channel. In Section III, we address the resource allocation problem with power control and known channel statistics. In Section IV, we consider the same problem without power control and channel statistics. Finally, we give our concluding remarks in Section V.

Regarding the notation, we denote by $x_{i}$ the $i$-th component of a vector $\boldsymbol{x}$. A vector $\boldsymbol{x}$ is positive when $x_{i}>0$ for all components $i$ of $\boldsymbol{x}$. We denote the nonnegative orthant by $\mathbb{R}_{+}^{n}$, i.e., $\mathbb{R}_{+}^{n}=\left\{\boldsymbol{x} \in \mathbb{R}^{n} \mid \boldsymbol{x} \geq 0\right\}$. We write $\boldsymbol{x}^{\prime}$ to denote the transpose of a vector $\boldsymbol{x}$.

\section{SYSTEM MODEL}

We consider $M$ transmitters sharing the same media to communicate to a single receiver. We model the channel as a Gaussian multiple access channel with flat fading effects

$$
Y(n)=\sum_{i=1}^{M} \sqrt{H_{i}(n)} X_{i}(n)+Z(n),
$$

where $X_{i}(n)$ and $H_{i}(n)$ are the transmitted waveform and the fading process of the $i$-th transmitter, respectively, and $Z(n)$ 
is white Gaussian noise with variance $N_{0}$. We assume that the fading processes of all transmitters are jointly stationary and ergodic, and the stationary distribution of the fading process has continuous density. We also assume that all the transmitters and the receiver have instant access to channel state information. In practice, the receiver measures the channels and feeds back the channel information to the transmitters. The implicit assumption in this model is that the channel variations are much slower than the data rate, so that the channel can be measured accurately at the receiver and the amount of feedback bits is negligible compared to that of transmitting information.

First, consider the non-fading case where the channel gains are fixed. The capacity region of the Gaussian multiple-access channel with no power control is described as follows [10]

$$
\begin{gathered}
C_{g}(\boldsymbol{P}, \boldsymbol{h})=\left\{\boldsymbol{R} \in \mathbb{R}_{+}^{M}: \sum_{i \in S} R_{i} \leq C\left(\sum_{i \in S} h_{i} P_{i}, N_{0}\right),\right. \\
\text { for all } S \subseteq \mathcal{M}=\{1, \ldots, M\}\},
\end{gathered}
$$

where $P_{i}$ and $R_{i}$ are the $i$-th transmitter's power and rate, respectively. $C(P, N)$ denotes Shannon's formula for the capacity of AWGN channel given by

$$
C(P, N)=\frac{1}{2} \log \left(1+\frac{P}{N}\right) \text { nats. }
$$

For a multiple-access channel with fading, but fixed transmission powers $P_{i}$, the throughput capacity region is given by averaging the instantaneous capacity regions with respect to the fading process [11],

$$
\begin{gathered}
C_{a}(\boldsymbol{P})=\left\{\boldsymbol{R} \in \mathbb{R}_{+}^{M}: \sum_{i \in S} R_{i} \leq \mathbb{E}_{\boldsymbol{H}}\left[C\left(\sum_{i \in S} H_{i} P_{i}, N_{0}\right)\right],\right. \\
\text { for all } S \subseteq \mathcal{M}\}
\end{gathered}
$$

where $\boldsymbol{H}$ is a random vector with the stationary distribution of the fading process.

A power control policy $\mathcal{P}$ is a map from any given fading state $\boldsymbol{h}$ to $\mathcal{P}(\boldsymbol{h})=\left(\mathcal{P}_{1}(\boldsymbol{h}), \ldots, \mathcal{P}_{M}(\boldsymbol{h})\right)$, the powers allocated to the transmitters. Similarly, we can define the rate allocation policy, $\mathcal{R}$, as a map from the fading state $\boldsymbol{h}$ to the transmission rates, $\mathcal{R}(\boldsymbol{h})$. For any given power-control policy $\mathcal{P}$, the capacity region follows from (4) as

$$
\begin{aligned}
C_{f}(\mathcal{P})= & \left\{\boldsymbol{R} \in \mathbb{R}_{+}^{M}: \sum_{i \in S} R_{i} \leq\right. \\
& \left.\mathbb{E}_{\boldsymbol{H}}\left[C\left(\sum_{i \in S} H_{i} \mathcal{P}_{i}(\boldsymbol{H}), N_{0}\right)\right], \text { for all } S \subseteq \mathcal{M}\right\} .
\end{aligned}
$$

Tse et al. [4] have shown that the throughput capacity of a multiple access fading channel is given by

$$
C(\overline{\boldsymbol{P}})=\bigcup_{\mathcal{P} \in \mathcal{G}} C_{f}(\mathcal{P}),
$$

where $\mathcal{G}=\left\{\mathcal{P}: \mathbb{E}_{\boldsymbol{H}}\left[\mathcal{P}_{i}(\boldsymbol{H})\right] \leq \bar{P}_{i}\right.$, for all $\left.i\right\}$ is the set of all power control policies satisfying the average power constraint. Let us define the notion of boundary or dominant face for any of the capacity regions defined above.
Definition 1: The dominant face or boundary of a capacity region, denoted by $\mathcal{F}(\cdot)$, is defined as the set of all $M$-tuples in the capacity region such that no component can be increased without decreasing others while remaining in the capacity region.

\section{Rate Allocation with Power Control}

In this section, we assume that the channel statistics are known a priori. The goal of optimal resource allocation is to find feasible rate and power allocation policies denoted by $\mathcal{R}^{*}$ and $\mathcal{P}^{*}$, respectively, such that $\mathcal{R}^{*}(\boldsymbol{H}) \in C_{g}\left(\mathcal{P}^{*}(\boldsymbol{H}), \boldsymbol{H}\right)$, and $\mathcal{P}^{*} \in \mathcal{G}$. Moreover,

$$
\begin{aligned}
& \mathbb{E}_{\boldsymbol{H}}\left[\mathcal{R}^{*}(\boldsymbol{H})\right]=\boldsymbol{R}^{*}=\operatorname{argmax} u(\boldsymbol{R}) \\
& \text { subject to } \boldsymbol{R} \in C(\overline{\boldsymbol{P}})
\end{aligned}
$$

where $u(\cdot)$ is a given utility function and is assumed to be a continuously differentiable concave function of $\boldsymbol{R}$, and monotonically increasing in each component $R_{i}$ for all $i$.

For the case of a linear utility function, i.e., $u(\boldsymbol{R})=\boldsymbol{\mu}^{\prime} \boldsymbol{R}$ for some $\boldsymbol{\mu} \in \mathbb{R}_{+}^{M}$, Tse et al. [4] have shown that the optimal rate and power allocation policies are given by the optimal solution to a linear program, i.e.,

$$
\begin{aligned}
\left(\mathcal{R}^{*}(\boldsymbol{h}), \mathcal{P}^{*}(\boldsymbol{h})\right)= & \underset{\boldsymbol{r}, \boldsymbol{p}}{\arg \max _{\boldsymbol{n}}}\left(\boldsymbol{\mu}^{\prime} \boldsymbol{r}-\boldsymbol{\lambda}^{\prime} \boldsymbol{p}\right) \\
& \text { subject to } \quad \boldsymbol{r} \in C_{g}(\boldsymbol{h}, \boldsymbol{p}),
\end{aligned}
$$

where $\boldsymbol{h}$ is the channel state realization, and $\boldsymbol{\lambda} \in \mathbb{R}_{+}^{M}$ is a Lagrange multiplier satisfying the average power constraint, i.e., $\boldsymbol{\lambda}$ is the unique solution of the following equations

$$
\begin{aligned}
\int_{0}^{\infty} & \frac{1}{h} \int_{\frac{2 \lambda_{i}\left(N_{0}+z\right)}{\mu_{i}}}^{\infty} \\
& \prod_{k \neq i} F_{k}\left(\frac{2 \lambda_{k} h\left(N_{0}+z\right)}{2 \lambda_{i}\left(N_{0}+z\right)+\left(\mu_{k}-\mu_{i}\right) h}\right) f_{i}(h) \mathrm{d} h \mathrm{~d} z=\bar{P}_{i}
\end{aligned}
$$

where $F_{k}$ and $f_{k}$ are cumulative distribution function (CDF) and probability density function (PDF) of the stationary distribution of the channel state process for transmitter $k$, respectively.

Exploiting the polymatroid structure of the capacity region, problem (7) can be solved by a simple greedy algorithm (see Lemma 3.2 of [4]). It is also shown in [4] that for positive $\boldsymbol{\mu}$ the optimal solution, $\boldsymbol{R}^{*}$, to the problem in (6) is uniquely obtained. Given the distribution of channel state process, denoted by $F_{k}$ and $f_{k}$, we have

$$
\begin{aligned}
R_{i}^{*}(\boldsymbol{\mu})= & \int_{0}^{\infty} \frac{1}{2\left(N_{0}+z\right)} \int_{\frac{2 \lambda_{i}\left(N_{0}+z\right)}{\mu_{i}}}^{\infty} \\
& \prod_{k \neq i} F_{k}\left(\frac{2 \lambda_{k} h\left(N_{0}+z\right)}{2 \lambda_{i}\left(N_{0}+z\right)+\left(\mu_{k}-\mu_{i}\right) h}\right) f_{i}(h) \mathrm{d} h \mathrm{~d} z,
\end{aligned}
$$

The uniqueness of $\boldsymbol{R}^{*}$ follows from the fact that the stationary distribution of the fading process has continuous density [4]. It is worth mentioning that (9) parametrically describes the 
boundary of the capacity region, and hence, there is a one-toone correspondence between the boundary of $C(\overline{\boldsymbol{P}})$ and the positive vectors $\boldsymbol{\mu}$ with unit norm.

Now consider a general concave utility function. We use an iterative method to compute the optimal solution, $\boldsymbol{R}^{*}$, of problem (6). Note that by monotonicity of the utility function, $\boldsymbol{R}^{*}$ always lies on the boundary of the capacity region, $C(\overline{\boldsymbol{P}})$. Once $\boldsymbol{R}^{*}$ is known, then in view of one-to-one correspondence between the boundary of $C(\overline{\boldsymbol{P}})$ and the positive vectors $\boldsymbol{\mu}$, there exist a positive vector $\boldsymbol{\mu}^{*}$ such that

$$
\boldsymbol{R}^{*}=\operatorname{argmax} \quad\left(\boldsymbol{\mu}^{*}\right)^{\prime} \boldsymbol{R} \quad \text { subject to } \quad \boldsymbol{R} \in C(\overline{\boldsymbol{P}}) .
$$

Therefore the optimal rate and power allocation policies can be obtained by using the greedy policies of Tse et al. [4] for the linear utility function, $u(\boldsymbol{R})=\left(\boldsymbol{\mu}^{*}\right)^{\prime} \boldsymbol{R}$.

We use the conditional gradient method [12] in order to iteratively compute the optimal solution of problem (6). The $k$-th iteration of the method is given by

$$
\boldsymbol{R}^{k+1}=\boldsymbol{R}^{k}+\alpha^{k}\left(\overline{\boldsymbol{R}}^{k}-\boldsymbol{R}^{k}\right),
$$

where $\alpha^{k}$ is the stepsize and $\overline{\boldsymbol{R}}^{k}$ is obtained as

$$
\overline{\boldsymbol{R}}^{k} \in \underset{\boldsymbol{R} \in C(\overline{\boldsymbol{P}})}{\arg \max }\left(\nabla u\left(\boldsymbol{R}^{k}\right)^{\prime}\left(\boldsymbol{R}-\boldsymbol{R}^{k}\right)\right) .
$$

Since the utility function is monotonically increasing, the gradient vector is always positive and, hence, the unique optimal solution to the above sub-problem is obtained by (9), in which $\boldsymbol{\mu}$ is replaced by $\nabla u\left(\boldsymbol{R}^{k}\right)$. By concavity of the utility function and convexity of the capacity region, the iteration (11) will converge to the optimal solution of (6) for appropriate stepsize selection rules such as Armijo rule or limited maximization rule (c.f. [12] pp. 220-222).

Note that our goal is to determine rate and power allocation policies. Finding $\boldsymbol{R}^{*}$ allows us to determine such policies by the greedy policy in (7) for $\boldsymbol{\mu}^{*}=\nabla u\left(\boldsymbol{R}^{*}\right)$. It is worth mentioning that all the computations for obtaining $\boldsymbol{R}^{*}$ are performed once in the setup of the communication session. So the convergence rate of the conditional gradient method is generally not of critical importance.

\section{Rate Allocation without Power Control}

In this section we assume that the channel statistics are not known and that the transmission powers are fixed to $\boldsymbol{P}$. In practice, this scenario occurs when the transmission power may be limited owing to environmental limitations such as human presence, or limitations of the hardware.

The capacity region of the multiple access channel for this scenario is a polyhedron and is given by (4). Similarly to the previous case, the optimal rate allocation policy, $\mathcal{R}^{*}(\cdot)$, is such that $\mathcal{R}^{*}(\boldsymbol{H}) \in C_{g}(\boldsymbol{P}, \boldsymbol{H})$, and

$$
\begin{aligned}
& \mathbb{E}_{\boldsymbol{H}}\left[\mathcal{R}^{*}(\boldsymbol{H})\right]=\boldsymbol{R}^{*} \quad \underset{\text { subject to }}{\operatorname{argmax}} \quad u(\boldsymbol{R}) \\
& \boldsymbol{R} \in C_{a}(\boldsymbol{P}) .
\end{aligned}
$$

It is worth mentioning that the approach used to find the optimal resource allocation policies for the previous case does not apply to this scenario, because $C_{g}(\boldsymbol{P}, \boldsymbol{h})$ is a polyhedron and hence, the uniqueness property of $\boldsymbol{R}^{*}$ for any positive vector $\boldsymbol{\mu}$ does not hold anymore.

Here we present a greedy rate allocation policy and compare its performance with the unknown optimal policy. The performance of a particular rate allocation policy is defined as the utility at the average rate achieved by that policy. The greedy policy, denoted by $\overline{\mathcal{R}}(\cdot)$, optimizes the utility function for any channel realization. i.e.,

$$
\overline{\mathcal{R}}(\boldsymbol{h})=\operatorname{argmax}_{\boldsymbol{R} \in C_{g}(\boldsymbol{P}, \boldsymbol{h})} \quad u(\boldsymbol{R}) .
$$

Consider the following relations

$$
\begin{aligned}
\mathbb{E}_{\boldsymbol{H}}\left[u\left(\mathcal{R}^{*}(\boldsymbol{H})\right)\right] & \leq \mathbb{E}_{\boldsymbol{H}}[u(\overline{\mathcal{R}}(\boldsymbol{H}))] \\
& \leq u\left(\mathbb{E}_{\boldsymbol{H}}[\overline{\mathcal{R}}(\boldsymbol{H})]\right) \\
& \leq u\left(\mathbb{E}_{\boldsymbol{H}}\left[\mathcal{R}^{*}(\boldsymbol{H})\right]\right)
\end{aligned}
$$

where the second inequality follows from the Jensen's inequality by concavity of the utility function.

In the case of a linear utility function we have $u\left(\mathbb{E}_{\boldsymbol{H}}\left[\mathcal{R}^{*}(\boldsymbol{H})\right]\right)=\mathbb{E}_{\boldsymbol{H}}\left[u\left(\mathcal{R}^{*}(\boldsymbol{H})\right)\right]$, so equality holds throughout in (15) and $\overline{\mathcal{R}}(\cdot)$ is indeed the optimal rate allocation policy. For nonlinear utility functions, the greedy policy can be strictly suboptimal.

However, the greedy policy is not arbitrarily worse than the optimal one. In view of (15), we can bound the performance difference, $u\left(\boldsymbol{R}^{*}\right)-u\left(\mathbb{E}_{\boldsymbol{H}}[\overline{\mathcal{R}}(\boldsymbol{H})]\right)$, by bounding $\left|u\left(\mathbb{E}_{\boldsymbol{H}}\left[\mathcal{R}^{*}(\boldsymbol{H})\right]\right)-u\left(\mathbb{E}_{\boldsymbol{H}}[\overline{\mathcal{R}}(\boldsymbol{H})]\right)\right|$ or $\mid u\left(\mathbb{E}_{\boldsymbol{H}}\left[\mathcal{R}^{*}(\boldsymbol{H})\right]\right)-$ $\mathbb{E}_{\boldsymbol{H}}\left[u\left(\mathcal{R}^{*}(\boldsymbol{H})\right)\right] \mid$ from above. We show that the first bound goes to zero as the channel variations become small and the second bound vanishes as the utility function tends to have a more linear structure.

Before stating the main theorems, let us introduce some useful definitions and lemmas.

Definition 2: Let $Q$ be a polyhedron described by a set of linear constraints, i.e.,

$$
Q=\left\{\boldsymbol{x} \in \mathbb{R}^{n}: A \boldsymbol{x} \leq \boldsymbol{b}\right\}
$$

Define the expansion of $Q$ by $\delta$, denoted by $\mathcal{E}_{\delta}(Q)$, as the polyhedron obtained by relaxing all the constraints in (16), i.e., $\mathcal{E}_{\delta}(Q)=\left\{\boldsymbol{x} \in \mathbb{R}^{n}: A \boldsymbol{x} \leq \boldsymbol{b}+\delta \mathbf{1}\right\}$, where $\mathbf{1}$ is the vector of all ones.

Definition 3: Let $X$ and $Y$ be two polyhedra described by a set of linear constraints. Let $\mathcal{E}_{d}(X)$ be an expansion of $X$ by relaxing its constraints by $d$. The distance $d_{H}(X, Y)$ between $X$ and $Y$ is defined as the minimum scalar $d$ such that $X \subseteq$ $\mathcal{E}_{d}(Y)$ and $Y \subseteq \mathcal{E}_{d}(X)$.

Lemma 1 extends Chebychev's inequality for capacity regions. It states that the time varying capacity region does not deviate much from its mean with high probability.

Lemma 1: Let $\boldsymbol{H}$ be a random vector with the stationary distribution of the fading process with mean $\overline{\boldsymbol{H}}$ and covariance matrix $K$. Then

$$
\boldsymbol{P r}\left\{d_{H}\left(C_{g}(\boldsymbol{P}, \boldsymbol{H}), C_{a}(\boldsymbol{P})\right)>\delta\right\} \leq \frac{\sigma_{H}^{2}}{\delta^{2}},
$$


where $\sigma_{H}^{2}$ is defined as

$$
\begin{aligned}
& \sigma_{H}^{2} \triangleq \frac{1}{4} \sum_{S \subseteq\{1, \ldots, M\}} \boldsymbol{\Gamma}_{S}^{\prime} K \boldsymbol{\Gamma}_{S}(1+ \\
& \left.\left[\left(1+\boldsymbol{\Gamma}_{S}^{\prime} \overline{\boldsymbol{H}}\right)\left(\sqrt{2 \log \left(1+\boldsymbol{\Gamma}_{S}^{\prime} \overline{\boldsymbol{H}}\right)}-\frac{\sqrt{\boldsymbol{\Gamma}_{S}^{\prime} K \boldsymbol{\Gamma}_{S}}}{2}\right)\right]^{2}\right),
\end{aligned}
$$

where

$$
\left(\boldsymbol{\Gamma}_{S}\right)_{i}= \begin{cases}\frac{P_{i}}{N_{0}}, & i \in S \\ 0, & \text { otherwise. }\end{cases}
$$

Proof: Define random variables $Y_{S}$ and $Z_{S}$ as the following:

$Y_{S}=\frac{1}{2} \log \left(1+\sum_{i \in S} \frac{H_{i} P_{i}}{N_{0}}\right)=\frac{1}{2} \log \left(1+Z_{S}\right), \quad$ for all $S \subseteq \mathcal{M}$.

The facet defining constraints of $C_{g}(\boldsymbol{P}, \boldsymbol{H})$ and $C_{a}(\boldsymbol{P})$ are of the form of $\sum_{i \in S} R_{i} \leq Y_{S}$ and $\sum_{i \in S} R_{i} \leq$ $\mathbb{E}\left[Y_{S}\right]$, respectively. Hence, by Definition 3 , we have $d_{H}\left(C_{g}(\boldsymbol{P}, \boldsymbol{H}), C_{a}(\boldsymbol{P})\right)>\delta$ if and only if $\left|Y_{S}-\mathbb{E}\left[Y_{S}\right]\right|>\delta$, for all $S \subseteq \mathcal{M}$. After some manipulations, the following relations can be verified by employing Chebyshev's inequality:

$$
\begin{aligned}
\operatorname{Pr} & \left\{d_{H}\left(C_{g}(\boldsymbol{P}, \boldsymbol{H}), C_{a}(\boldsymbol{P})\right)>\delta\right\} \\
& =\operatorname{Pr}\left\{\max _{S}\left|Y_{S}-\mathbb{E}\left[Y_{S}\right]\right|>\delta\right\} \\
& \leq \frac{1}{\delta^{2}} \sum_{S \subseteq \mathcal{M}} \sigma_{Y_{S}}^{2}
\end{aligned}
$$

where $\sigma_{Y_{S}}^{2}$ denotes variance of $Y_{s}$, and can be bounded from above as follows (c.f. Appendix II, [13])

$$
\sigma_{Y_{S}}^{2} \leq \frac{\sigma_{Z_{S}}^{2}}{4}\left(1+\left[\left(1+\bar{Z}_{S}\right)\left(\sqrt{2 \log \left(1+\bar{Z}_{S}\right)}-\frac{\sigma_{Z_{S}}}{2}\right)\right]^{2}\right)
$$

where

$$
\begin{gathered}
\bar{Z}_{S}=\mathbb{E}\left[\sum_{i \in S} H_{i} P_{i}\right]=\boldsymbol{\Gamma}_{S}^{\prime} \overline{\boldsymbol{H}} \\
\sigma_{Z_{S}}^{2}=\operatorname{var}\left(\sum_{i \in S} H_{i} P_{i}\right)=\boldsymbol{\Gamma}_{S}^{\prime} K \boldsymbol{\Gamma}_{S} .
\end{gathered}
$$

The desired result is concluded by substituting $\bar{Z}_{S}$ and $\sigma_{Z_{S}}^{2}$ in (22) and combing the result with (21).

The system parameter $\sigma_{H}^{2}$ in Lemma 1 is proportional to channel variations, and we expect it to vanish for small channel variations. The following lemma ensures that the distance between the optimal solutions of the utility maximization problem over two regions is small, provided that the regions are close to each other.

Lemma 2: Let $\boldsymbol{R}_{1}^{*}$ and $\boldsymbol{R}_{2}^{*}$ be the optimal solution of maximizing the utility over $C_{g}\left(\boldsymbol{P}, \boldsymbol{H}_{1}\right)$ and $C_{g}\left(\boldsymbol{P}, \boldsymbol{H}_{2}\right)$, respectively. If there exist positive scalars $A$ and $B$ such that

$$
\begin{array}{ccl}
\left|u\left(\boldsymbol{R}_{1}\right)-u\left(\boldsymbol{R}_{2}\right)\right| & \leq & B\left\|\boldsymbol{R}_{1}-\boldsymbol{R}_{2}\right\|, \\
& \text { for all } & \boldsymbol{R}_{i} \in \mathcal{F}\left(C_{g}\left(\boldsymbol{P}, \boldsymbol{H}_{i}\right)\right), \quad i=1,2 . \\
\left|u\left(\boldsymbol{R}_{i}^{*}\right)-u\left(\boldsymbol{R}_{i}\right)\right| & \geq & A\left\|\boldsymbol{R}_{i}^{*}-\boldsymbol{R}_{i}\right\|^{2}, \\
& \text { for all } & \boldsymbol{R}_{i} \in C_{g}\left(\boldsymbol{P}, \boldsymbol{H}_{i}\right), \quad i=1,2,
\end{array}
$$

and moreover if

$$
d_{H}\left(C_{g}\left(\boldsymbol{P}, \boldsymbol{H}_{1}\right), C_{g}\left(\boldsymbol{P}, \boldsymbol{H}_{2}\right)\right) \leq \delta
$$

then, we have

$$
\left\|\boldsymbol{R}_{1}^{*}-\boldsymbol{R}_{2}^{*}\right\| \leq \delta^{\frac{1}{2}}\left[\delta^{\frac{1}{2}}+\left(\frac{B}{A}\right)^{\frac{1}{2}}\right]
$$

Proof: Without loss of generality assume that $u\left(\boldsymbol{R}_{2}^{*}\right) \geq$ $u\left(\boldsymbol{R}_{1}^{*}\right)$. To simplify the notations for capacity regions, let $C_{1}=$ $C_{g}\left(\boldsymbol{P}, \boldsymbol{H}_{1}\right)$ be a polymatroid, i.e.,

$$
C_{1}=\left\{\boldsymbol{R} \in \mathbb{R}_{+}^{M}: \sum_{i \in S} R_{i} \leq f(S), \text { for all } S \subseteq \mathcal{M}\right\},
$$

for some submodular function $f(S)$, and let $C_{2}$ be an expansion of $C_{1}$ by $\delta$ as defined in Definition 2. We first show that for every $\boldsymbol{R} \in \mathcal{F}\left(C_{2}\right)$, there exists a vector $\boldsymbol{R}^{\prime} \in \mathcal{F}\left(C_{1}\right)$ such that $\left\|\boldsymbol{R}-\boldsymbol{R}^{\prime}\right\| \leq \delta$, where $\mathcal{F}(\cdot)$ denotes the dominant face of a capacity region as in Definition 1 .

Assume $R$ is a vertex of $C_{2}$. Then the polymatroid structure of $C_{2}$ implies that $R$ is the intersection of $M$ constraints corresponding to a chain of subsets of $\mathcal{M}$. Hence, there is some $k \in \mathcal{M}$ such that $R_{k}=f(\{k\})+\delta$. Choose $\boldsymbol{R}^{\prime}$ as follows

$$
R_{i}^{\prime}= \begin{cases}R_{i}-\delta, & i=k \\ R_{i}, & \text { otherwise. }\end{cases}
$$

$\boldsymbol{R}^{\prime}$ is obviously in a $\delta$-neighborhood of $\boldsymbol{R}$. Moreover, the constraint corresponding to the set $\mathcal{M}$ is active for $\boldsymbol{R}^{\prime}$, so we just need to show that $R^{\prime}$ is feasible in order to prove that it is on the dominant face. First, let us consider the sets $S$ that contain $k$. We have

$$
\sum_{i \in S} R_{i}^{\prime}=\sum_{i \in S} R_{i}-\delta \leq f(S) .
$$

Second, consider the case that $k \notin S$.

$$
\begin{aligned}
\sum_{i \in S} R_{i}^{\prime} & =\sum_{i \in S \cup\{k\}} R_{i}^{\prime}-R_{k}+\delta \\
& \leq f(S \cup\{k\})+\delta-R_{k} \\
& \leq f(S)+f(\{k\})+\delta-R_{l} \\
& =f(S) .
\end{aligned}
$$

where the first inequality come from (27), and the second inequality is valid because of the submodularity of the function $f(\cdot)$.

The previous argument establishes that the claim is true for each vertex $\boldsymbol{R}_{j}$ of the dominant face. But every other point $\boldsymbol{R}$ on the dominant face can be represented as a convex combination of the vertices, i.e.,

$$
\boldsymbol{R}=\sum_{j} \alpha_{j} \boldsymbol{R}_{j}, \quad \sum_{j} \alpha_{j}=1, \alpha_{j} \geq 0 .
$$


Using the convexity of the norm function, it is quite straightforward to show that the desired $\boldsymbol{R}^{\prime}$ is given by

$$
\boldsymbol{R}^{\prime}=\sum_{j} \alpha_{j} \boldsymbol{R}_{j}^{\prime},
$$

where $\boldsymbol{R}_{j}^{\prime}$ is obtained for each $\boldsymbol{R}_{j}$ in the same manner as in (26).

So we have verified that there exists a point, $R$, on the dominant face of $C_{1}=C_{g}\left(\boldsymbol{P}, \boldsymbol{H}_{1}\right)$ such that $\left\|\boldsymbol{R}_{2}^{*}-\boldsymbol{R}\right\| \leq \delta$. By monotonicity of the utility function the optimal solution $\boldsymbol{R}_{2}^{*}$ lies on the dominant face of $C_{g}\left(\boldsymbol{P}, \boldsymbol{H}_{2}\right)$, hence, from the hypothesis and the fact that $u\left(\boldsymbol{R}_{2}^{*}\right) \geq u\left(\boldsymbol{R}_{1}^{*}\right) \geq u(\boldsymbol{R})$, we conclude

$$
u\left(\boldsymbol{R}_{2}^{*}\right)-u(\boldsymbol{R})=\left|u\left(\boldsymbol{R}_{2}^{*}\right)-u(\boldsymbol{R})\right| \leq B\left\|\boldsymbol{R}_{2}^{*}-\boldsymbol{R}\right\| \leq B \delta .
$$

Now suppose that $\left\|\boldsymbol{R}_{1}^{*}-\boldsymbol{R}\right\|>\left(\frac{B}{A} \delta\right)^{\frac{1}{2}}$. By the hypothesis in (23) we can write

$$
u\left(\boldsymbol{R}_{1}^{*}\right)-u(\boldsymbol{R})=\left|u\left(\boldsymbol{R}_{1}^{*}\right)-u(\boldsymbol{R})\right|>B \delta .
$$

By subtracting (28) from (29) we obtain $u\left(\boldsymbol{R}_{2}^{*}\right)<u\left(\boldsymbol{R}_{1}^{*}\right)$ which is a contradiction. Therefore, $\left\|\boldsymbol{R}_{1}^{*}-\boldsymbol{R}\right\| \leq\left(\frac{B}{A} \delta\right)^{\frac{1}{2}}$, and the desired result follows immediately by invoking the triangle inequality.

The following theorem combines the results of the above lemmas to obtain a bound on the performance difference of the greedy and optimal policy.

Theorem 1: Let $\boldsymbol{R}^{*}$ be the optimal solution to (13), and $\overline{\mathcal{R}}(\cdot)$ the greedy rate allocation policy as defined in (14) for a non-negative concave utility function $u(\cdot)$. Then for every $\epsilon \in(0,1]$,

$$
\begin{aligned}
& u\left(\boldsymbol{R}^{*}\right)-u\left(\mathbb{E}_{\boldsymbol{H}}[\overline{\mathcal{R}}(\boldsymbol{H})]\right) \leq \epsilon u\left(\boldsymbol{R}^{*}\right)+(1-\epsilon) B(\epsilon) \\
& {\left[\left(\frac{\sigma_{H}}{\sqrt{\epsilon}}\right)^{\frac{1}{2}}+\left(\frac{B(\epsilon)}{A(\epsilon)}\right)^{\frac{1}{2}}\right]\left(\frac{\sigma_{H}}{\sqrt{\epsilon}}\right)^{\frac{1}{2}}, }
\end{aligned}
$$

where $B(\epsilon)$ and $A(\epsilon)$ are positive functions of $\epsilon$, such that for all $\boldsymbol{H}$ with $\left.d_{H}\left(C_{g}(\boldsymbol{P}, \boldsymbol{H}), C_{a}(\boldsymbol{P})\right)\right) \leq \frac{\sigma_{H}}{\sqrt{\epsilon}}$, they satisfy the following conditions.

$$
\begin{aligned}
\left|u\left(\boldsymbol{R}_{\boldsymbol{a}}\right)-u\left(\boldsymbol{R}_{\boldsymbol{g}}\right)\right| \leq & B(\epsilon)\left\|\boldsymbol{R}_{\boldsymbol{a}}-\boldsymbol{R}_{\boldsymbol{g}}\right\| \\
& \text { for all } \boldsymbol{R}_{\boldsymbol{a}} \in \mathcal{F}\left(C_{a}(\boldsymbol{P})\right), \\
& \text { for all } \boldsymbol{R}_{\boldsymbol{g}} \in \mathcal{F}\left(C_{g}(\boldsymbol{P}, \boldsymbol{H})\right), \\
|u(\overline{\mathcal{R}}(\boldsymbol{H}))-u(\boldsymbol{R})| \geq & A(\epsilon)\|\overline{\mathcal{R}}(\boldsymbol{H})-\boldsymbol{R}\|^{2} \\
& \text { for all } \boldsymbol{R} \in C_{g}(\boldsymbol{P}, \boldsymbol{H}) .
\end{aligned}
$$

Proof: Pick any $\epsilon \in(0,1]$. Define the event $\mathcal{V}$ as

$$
\left.d_{H}\left(C_{g}(\boldsymbol{P}, \boldsymbol{H}), C_{a}(\boldsymbol{P})\right)\right) \leq \frac{\sigma_{H}}{\sqrt{\epsilon}} .
$$

By Lemma 1, the probability of this event is at least $1-\epsilon$. Conditioned on $\mathcal{V}$, we have the following

$$
\begin{aligned}
\left|u\left(\boldsymbol{R}^{*}\right)-u(\overline{\mathcal{R}}(\boldsymbol{H}))\right| & \leq B(\epsilon)\left\|\overline{\mathcal{R}}(\boldsymbol{H})-\boldsymbol{R}^{*}\right\| \\
& \leq B(\epsilon)\left[\left(\frac{\sigma_{H}}{\sqrt{\epsilon}}\right)^{\frac{1}{2}}+\left(\frac{B(\epsilon)}{A(\epsilon)}\right)^{\frac{1}{2}}\right]\left(\frac{\sigma_{H}}{\sqrt{\epsilon}}\right)^{\frac{1}{2}},
\end{aligned}
$$

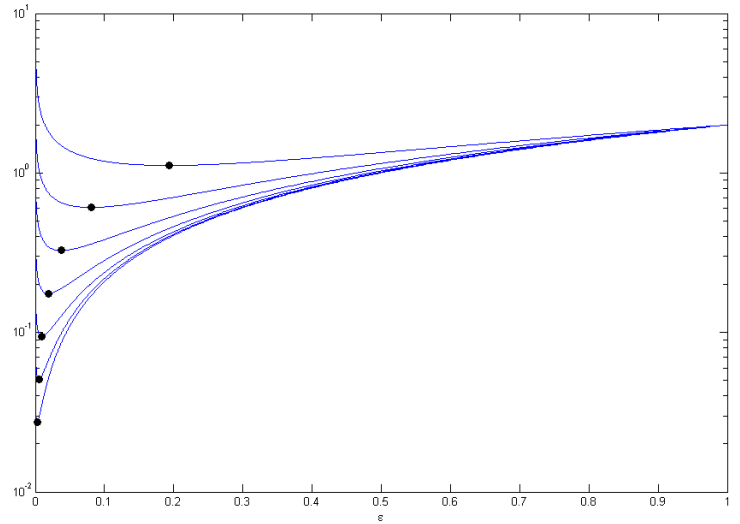

Fig. 1. Parameterized upperbound on performance difference between greedy and optimal policies.

where the first inequality follows from monotonicity of the utility function and (31). The second inequality is a direct result of applying Lemma 2.

Using Jensen's inequality as in (15) we can bound the lefthand side of (30) as follows

$$
\begin{aligned}
u\left(\boldsymbol{R}^{*}\right) & -u\left(\mathbb{E}_{\boldsymbol{H}}[\overline{\mathcal{R}}(\boldsymbol{H})]\right) \\
\leq & u\left(\boldsymbol{R}^{*}\right)-\mathbb{E}_{\boldsymbol{H}}[u(\overline{\mathcal{R}}(\boldsymbol{H}))] \\
\leq & u\left(\boldsymbol{R}^{*}\right)-(1-\epsilon) \mathbb{E}_{\boldsymbol{H}}[u(\overline{\mathcal{R}}(\boldsymbol{H})) \mid \mathcal{V}] \\
& -\operatorname{Pr}\left(\mathcal{V}^{c}\right) \mathbb{E}_{\boldsymbol{H}}\left[u(\overline{\mathcal{R}}(\boldsymbol{H})) \mid \mathcal{V}^{c}\right] \\
\leq & \epsilon u\left(\boldsymbol{R}^{*}\right)+(1-\epsilon) \mathbb{E}_{\boldsymbol{H}}\left[\left|u\left(\boldsymbol{R}^{*}\right)-u(\overline{\mathcal{R}}(\boldsymbol{H}))\right| \mid \mathcal{V}\right] .
\end{aligned}
$$

In the above relations, the second inequality follows from $\operatorname{Pr}(\mathcal{V}) \geq 1-\epsilon$, and the third inequality is obtained from non-negativity of the utility function after some manipulation. Replacing (33) in (34) gives the desired upperbound.

Theorem 1 provides a bound parameterized by $\epsilon$. For very small channel variations, $\sigma_{H}$ tends to zero, and we can choose $\epsilon$ proportional to $\sigma_{H}$ such that the bound in (30) approaches zero. Figure 1 illustrates the behavior of the parameterized bound provided in (30) for different values of $\sigma_{H}$. For each value of $\sigma_{H}$, the upperbound is minimized for a specific choice of $\epsilon$, which is illustrated as a dot in Figure 1. As demonstrated in the figure, for smaller channel variations tighter bound is achieved and the minimizer parameter decreases.

The next theorem provides another bound demonstrating the impact of the structure of the utility function on the performance of the greedy policy.

Theorem 2: Let $\boldsymbol{R}^{*}$ be the optimal solution to (13) for the non-negative utility function $u(\boldsymbol{R})$. Also let $\mathcal{R}^{*}(\cdot)$ and $\overline{\mathcal{R}}(\cdot)$ be the optimal and greedy rate allocation policies, respectively. Then for every $\epsilon \in(0,1]$,

$$
u\left(\boldsymbol{R}^{*}\right)-u\left(\mathbb{E}_{\boldsymbol{H}}[\overline{\mathcal{R}}(\boldsymbol{H})]\right) \leq \epsilon u\left(\boldsymbol{R}^{*}\right)+\frac{1}{2}(1-\epsilon) r(\epsilon)^{2} \Omega,
$$

where $\Omega$ satisfies the following

$$
\lambda_{\max }\left(-\nabla^{2} u(\boldsymbol{\xi})\right) \leq \Omega, \quad \text { for all } \boldsymbol{\xi},\left\|\boldsymbol{\xi}-\boldsymbol{R}^{*}\right\| \leq r(\epsilon),
$$


and $r(\epsilon)$ is given by

$$
\begin{aligned}
r(\epsilon) & =\sqrt{M} \frac{\sigma_{H}}{\sqrt{\epsilon}}+ \\
& {\left[\sum_{i=1}^{M} \mathbb{E}_{\boldsymbol{H}}\left[\frac{1}{2} \log \left(\frac{\left(1+H_{i} P_{i}\right)\left(1+\sum_{j \neq i} H_{j} P_{j}\right)}{1+\sum_{j=1}^{M} H_{j} P_{j}}\right)\right]^{2}\right]^{\frac{1}{2}} . }
\end{aligned}
$$

Proof: Pick any $\epsilon \in(0,1]$. Define the event $\mathcal{V}$ similarly to the proof of Theorem 1. Because of monotonicity of the utility function, we know that $\boldsymbol{R}^{*}$ lies on the dominant face of $C_{a}(\boldsymbol{P})$. Since the region $C_{a}(\boldsymbol{P})$ is the average of all regions $C_{g}(\boldsymbol{P}, \boldsymbol{H})$, the optimal policy $\mathcal{R}^{*}(\boldsymbol{H})$ should give a point on the dominant face of $C_{g}(\boldsymbol{P}, \boldsymbol{H})$, for almost all $\boldsymbol{H}$. Therefore, conditioned on $\mathcal{V}$, we can bound the set in which $\mathcal{R}^{*}(\boldsymbol{H})$ ranges, i.e., $\left\|\mathcal{R}^{*}(\boldsymbol{H})-\boldsymbol{R}^{*}\right\| \leq r(\epsilon)$, after some straightforward manipulations. Now let us write the Taylor expansion of the function $u(\cdot)$ at $\boldsymbol{R}^{*}$. We have

$$
\begin{aligned}
u(\boldsymbol{R})= & u\left(\boldsymbol{R}^{*}\right)+\nabla u\left(\boldsymbol{R}^{*}\right)^{\prime}\left(\boldsymbol{R}-\boldsymbol{R}^{*}\right) \\
& -\frac{1}{2}\left(\boldsymbol{R}-\boldsymbol{R}^{*}\right)^{\prime}\left(-\nabla^{2} u(\boldsymbol{\xi})\right)\left(\boldsymbol{R}-\boldsymbol{R}^{*}\right) \\
\geq & u\left(\boldsymbol{R}^{*}\right)+\nabla u\left(\boldsymbol{R}^{*}\right)^{\prime}\left(\boldsymbol{R}-\boldsymbol{R}^{*}\right) \\
& -\frac{1}{2}\left\|\boldsymbol{R}-\boldsymbol{R}^{*}\right\|^{2} \lambda_{\max }\left(-\nabla^{2} u(\boldsymbol{\xi})\right),
\end{aligned}
$$

for some $\boldsymbol{\xi},\left\|\boldsymbol{\xi}-\boldsymbol{R}^{*}\right\| \leq\left\|\boldsymbol{R}-\boldsymbol{R}^{*}\right\|$.

By replacing $\boldsymbol{R}$ by $\mathcal{R}^{*}(\boldsymbol{H})$ and conditioning on $\mathcal{V}$ we have the following

$$
\begin{aligned}
u\left(\mathcal{R}^{*}(\boldsymbol{H})\right) \geq & u\left(\boldsymbol{R}^{*}\right)+ \\
& \nabla u\left(\boldsymbol{R}^{*}\right)^{\prime}\left(\mathcal{R}^{*}(\boldsymbol{H})-\boldsymbol{R}^{*}\right)-\frac{1}{2} r(\epsilon)^{2} \Omega .
\end{aligned}
$$

Now we can bound the left-hand side of (35) by bounding the Jensen's difference $u\left(\boldsymbol{R}^{*}\right)-\mathbb{E}_{\boldsymbol{H}}\left[u\left(\mathcal{R}^{*}(\boldsymbol{H})\right)\right]$. After some manipulation similar to (34), we have

$$
\begin{aligned}
& u\left(\boldsymbol{R}^{*}\right)-u\left(\mathbb{E}_{\boldsymbol{H}}[\overline{\mathcal{R}}(\boldsymbol{H})]\right) \\
& \leq \quad u\left(\boldsymbol{R}^{*}\right)-\mathbb{E}_{\boldsymbol{H}}\left[u\left(\mathcal{R}^{*}(\boldsymbol{H})\right)\right] \\
& \leq \quad u\left(\boldsymbol{R}^{*}\right)-(1-\epsilon) \mathbb{E}_{\boldsymbol{H}}\left[u\left(\mathcal{R}^{*}(\boldsymbol{H})\right) \mid \mathcal{V}\right] \\
& \quad-\operatorname{Pr}\left(\mathcal{V}^{c}\right) \mathbb{E}_{\boldsymbol{H}}\left[u\left(\mathcal{R}^{*}(\boldsymbol{H})\right) \mid \mathcal{V}^{c}\right] \\
& \leq \quad u\left(\boldsymbol{R}^{*}\right)-(1-\epsilon)\left(u\left(\boldsymbol{R}^{*}\right)-\frac{1}{2} r(\epsilon)^{2} \Omega\right) \\
&= \epsilon u\left(\boldsymbol{R}^{*}\right)+\frac{1}{2}(1-\epsilon) r(\epsilon)^{2} \Omega .
\end{aligned}
$$

Similarly to Theorem 1, Theorem 2 provides a bound parameterized by $\epsilon$ which goes to zero for proper choice of $\epsilon$ as $\Omega$ becomes smaller and the utility function tends to have a more linear structure. The behavior of this parameterized upperbound is also similar to the one illustrated in Figure 1.

In summary, the performance difference between the greedy and the optimal policy is bounded from above by the minimum of the bounds provided by Theorem 1 and Theorem 2 .

\section{CONCLuSiON}

We addressed the problem of optimal resource allocation in a fading multiple access channel from an information theoretic point of view. We formulated the problem as a utility maximization problem for a more general class of utility functions.

We considered two different scenarios. First, we assumed that the transmitters have power control feature and the channel statistics are known a priori. In this case, the optimal rate and power allocation policies are obtained by greedily maximizing a properly defined linear utility function.

In the second scenario, power control and channel statistics are not available. In this case, the greedy policy is not optimal for nonlinear utility functions. However, we showed that its performance in terms of the utility is not arbitrarily worse compared to the optimal policy, by bounding their performance difference. The provided bound tends to zero as the channel variations become small or the utility function behaves more linearly.

The greedy policy may itself be computationally expensive. A computationally efficient algorithm can be employed to allocate rates close to the ones allocated by the greedy policy. This algorithm just takes one iteration of the gradient projection method at each time slot. Under slow fading conditions, it can be shown that this method tracks the greedy policy very closely, and its performance is close to the optimal policy.

\section{REFERENCES}

[1] X. Wang and G.B. Giannakis. Energy-efficient resource allocation in time division multiple-access over fading channels. Preprint, 2005.

[2] S.J. Oh, Z. Danlu, and K.M. Wasserman. Optimal resource allocation in multiservice CDMA networks. IEEE Transactions on Wireless Communications, 2(4):811-821, 2003.

[3] J.B. Kim and M.L. Honig. Resource allocation for multiple classes of DS-CDMA traffic. IEEE Transactions on Vehicular Technology, 49(2):506-519, 2000.

[4] D. Tse and S. Hanly. Multiaccess fading channels part I: Polymatroid structure, optimal resource allocation and throughput capacities. IEEE Transactions on Information Theory, 44(7):2796-2815, 1998.

[5] S. Shenker. Fundamental design issues for the future internet. IEEE Journal on Selected Areas in Communications, 13:1176-118, 1995.

[6] R. Srikant. Mathematics of Internet Congestion Control. Birkhauser, 2004.

[7] S. Vishwanath, S.A. Jafar, and A. Goldsmith. Optimum power and rate allocation strategies for multiple access fading channels. In Proceedings of IEEE VTC, 2001.

[8] D. Yu and J.M. Cioffi. Iterative water-filling for optimal resource allocation in OFDM multiple-access and broadcast channels. In Proceedings of IEEE GLOBECOM, 2006.

[9] K. Seong, R. Narasimhan, and J. Cioffi. Scheduling for fading multiple access channels with heterogeneous QoS constraints. In Proceedings of International Symposium on Information Theory, 2007.

[10] T.M. Cover and J.A. Thomas. Elements of Information Theory. John Wiley and Sons, Inc., New York, New York, 1991.

[11] S. Shamai and A.D. Wyner. Information theoretic considerations for symmetric, cellular, multiple-access fading channels part I. IEEE Transactions on Information Theory, 43(6):1877-1894, 1997.

[12] D.P. Bertsekas. Nonlinear Programming. Athena Scientific, Cambridge, Massachusetts, 1999.

[13] Ali ParandehGheibi. Resource allocation in multiple-access channels LIDS technical report, Massachusetts Institute of Technology, 2008. 